\title{
Magnetism in $\mathrm{NdMnO}_{3+\delta}$ Studied by the Single Crystal Neutron Diffraction
}

\author{
M. Mihalik JR. ${ }^{a, *}$, M. Mihalik ${ }^{a}$, S. MAŤA $\check{S}^{b}$, M. VAVRA ${ }^{a, c}$ \\ ${ }^{a}$ Institute of Experimental Physics SAS, Watsonova 47, 04001 Košice, Slovakia \\ ${ }^{b}$ Helmholtz-Zentrum Berlin, Hahn Meitner Platz 1, D-14109 Berlin, Germany \\ ${ }^{c}$ Institute of Chemistry, Faculty of Science, P. J. Šafárik University, Moyzesova 11, 04154 Košice, Slovakia
}

\begin{abstract}
We present the single crystal neutron diffraction experiment performed on the $\mathrm{NdMnO}_{3+\delta}$ compound. The aim of the experiment was to study/revisit the magnetic structure of the compound. We have confirmed the ordering of $\mathrm{Mn}$ sublattice below $T_{N}=85.5 \mathrm{~K}$ and confirmed that the Nd sublattice orders below $T_{1} \approx 20 \mathrm{~K}$ with Nd moments aligned parallel to the $b$-axis.
\end{abstract}

DOI: 10.12693/APhysPolA.126.284

PACS: 75.20.-g, 75.30.-m, 75.50.Gg

The $\mathrm{ReTO}_{3}$ compounds $(\mathrm{Re}=$ rare earth, $\mathrm{T}=\mathrm{Mn}, \mathrm{Fe})$ attract large attention due to their very interesting physical properties e. g. multiferroicity and ferroelectricity [1], compensation temperatures [2,3], promising application potential in the field of magnetocaloric effect at room temperature [4], nonlinear magnetooptical effects in the $\mathrm{THz}$ region and other physical properties.

Neutron powder diffraction (NPD) experiments performed on $\mathrm{NdMnO}_{3}$ revealed that the compound orders antiferromagnetically (ordering of $\mathrm{Mn}$ sublattice, spin arrangement $\left.\left(\mathrm{C}_{x} \mathrm{~F}_{y} 0\right)\right)$ at $T_{N}=82 \mathrm{~K}[5]$ and exhibits another transition which corresponds with additional anomaly in $\mathrm{AC}$ susceptibility around $T_{1} \approx 20 \mathrm{~K}$ [6]. The ordering at $T_{N}$ is ascribed to the superexchange between $\mathrm{Mn}$ ions mediated by oxygen atoms [2], while the description of the transition at $T_{1}$ remains controversial: One group of authors claim that Nd sublattice orders ferromagnetically (moments parallel to $c$-axis) at $T_{1}$ as a consequence of the polarization of $\mathrm{Nd}^{3+}$ ions by $\mathrm{Mn}$ ions (i.e. order-to-order magnetic phase transition) [5], while the other group of authors claim that Nd ions possess the ordered magnetic moment already below $T_{N}$ and effect at $T_{1}$ is the consequence of the segregation of the ferromagnetic clusters within the antiferromagnetic matrix [3]. It is also worth to mention that the interplay of the magnetic interactions in this type of system leads to an effect of negative magnetization and compensation temperatures if the compound is slightly non-stoichiometric $\left(\mathrm{NdMnO}_{3.11}[2,3]\right)$, or slightly doped $\left(\mathrm{NdMn}_{0.8} \mathrm{Fe}_{0.2} \mathrm{O}_{3}\right.$ [6], $\left.\mathrm{Nd}_{0.92} \mathrm{Ca}_{0.08} \mathrm{MnO}_{3}[7]\right)$. Since in this compound manganese ions may be in $3+$ or $4+$ oxidation state, the nonstoichiometry of the compound, which depends on the preparation route, is a common phenomenon $[2,7]$. In our paper we have focused on study of magnetic structure, which was derived from neutron diffraction experiment performed on a single crystal.

*corresponding author; e-mail: matmihalik@saske.sk
Single crystals of $\mathrm{NdMnO}_{3+\delta}$ were prepared by floating zone method in an optical mirror furnace. As starting materials we have used oxides of $\mathrm{MnO}_{2}, \mathrm{Nd}_{2} \mathrm{O}_{3}$ and $\mathrm{Fe}_{2} \mathrm{O}_{3}$ with $3 \mathrm{~N}$ purity, which were mixed in a stoichiometric ratio, cold pressed into rods and sintered at $1200^{\circ} \mathrm{C}$ for 12 hours in air. The floating zone experiments were performed using the 4-mirror optical furnace equipped with $1 \mathrm{~kW}$ halogen lamps. We have used pulling speed of $5 \mathrm{~mm} / \mathrm{h}$, rotation of both shafts $10 \mathrm{rpm}$ and flowing $(2 \mathrm{l} / \mathrm{min})$ air atmosphere. The quality of the grown single crystals was checked on E4 two axis neutron diffractometer (Helmholtz Zentrum Berlin). It was found that the crystal has only the moderate quality with several sub-grains miss-oriented by roughly $0.75^{\circ}$. The oxygen nonstoichiometry $(\delta)$ was estimated by the iodometric titration and has value of about $\delta=0.02$. The neutron diffraction experiment was performed on E4 diffractometer, using the standard Orange cryostat and neutron wavelength of $2.432 \AA$. During the experiment we have studied 21 different reflections in the temperature range $1.6-100 \mathrm{~K}$.

All observed reflections were indexed by integer $\mathrm{h}, \mathrm{k}$, 1 indexes. No change in $2 \theta$ and $\omega$ during the temperature scans was observed. This suggests that the magnetic unit cell is identical with the crystallographic unit cell [magnetic $\boldsymbol{k}$ vector equal to $\left(\begin{array}{lll}0 & 0 & 0\end{array}\right)$ ] and that the crystal structure of the material does not change. Integrated intensities of the studied reflections can be divided into three groups: i) intensity keeps constant value or changes only slightly in the whole temperature range (Fig. 1a), ii) intensity increases below $T_{N} \approx 80 \mathrm{~K}$ (Fig. $1 \mathrm{~b}$ ) and iii) intensity is constant at temperatures higher than $T \approx 30 \mathrm{~K}$, but at lower temperatures increases (Fig. 1c).

Neutron diffraction experiment performed on a single crystal revealed that reflections (010) (Fig. 1b) and (200) (Fig. 2) gain the intensity by cooling down below $80 \mathrm{~K}$. We assign this extra intensity to the ordering of Mn sublattice $\left(T_{N}=85.5(2) \mathrm{K}[6]\right)$. At $T_{N}$ we have observed no increase of the intensity on (002) reflection (Fig. 1c) within the experimental error. These results are completely in agreement with NPD data published by Muñoz 


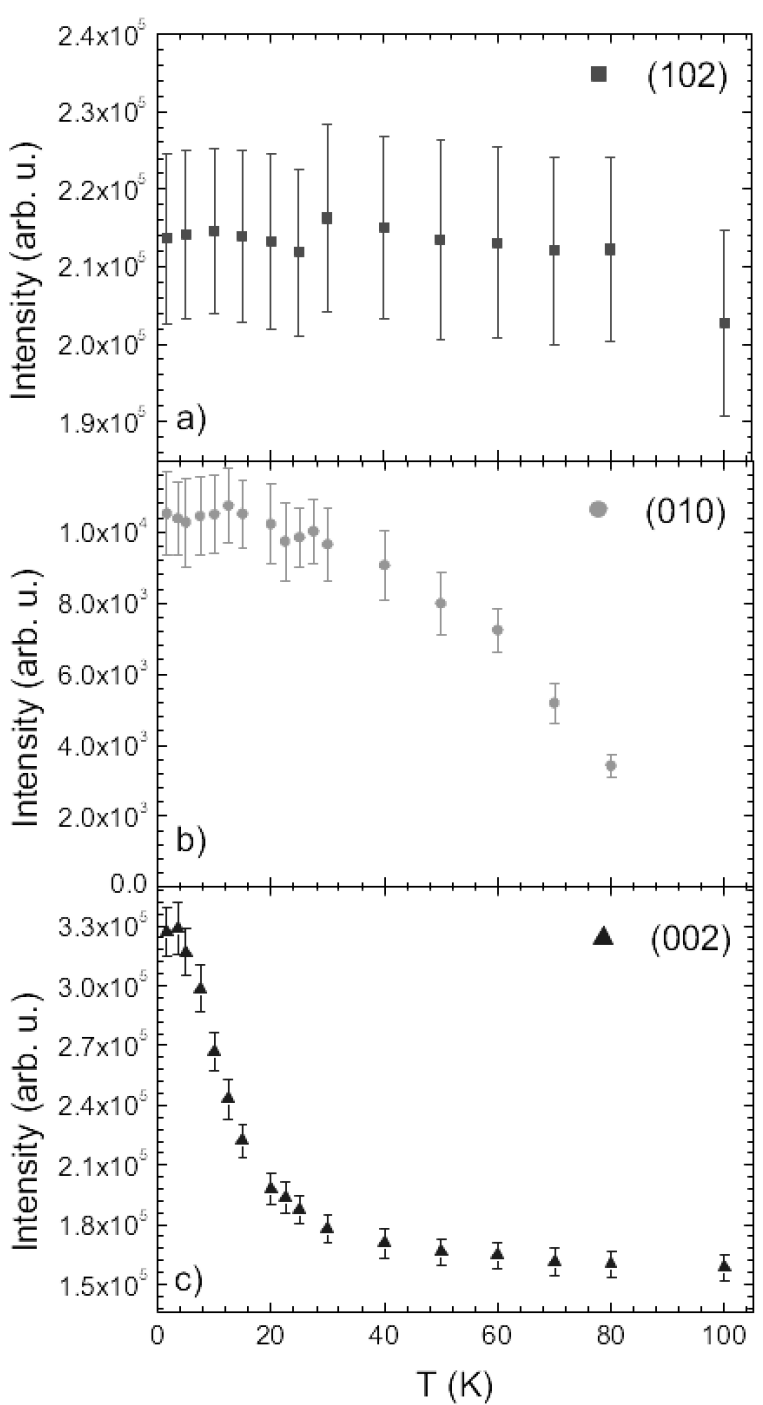

Fig. 1. Representative set of the observed reflections. The intensities are normalized to the same monitor. Note, that we index reflections using the space group Pnma; and lattice parameters: $a=5.838 \AA, b=$ $7.547 \AA, c=5.414 \AA$.

et al. [5]. Additionally, some reflections, like (002) (see Fig. 1c), or (200) (Fig. 2) gain intensity below $25-30 \mathrm{~K}$, which we ascribe to the order-to-order magnetic phase transition. Following Muñoz et al. [5] we estimate that the Nd sublattice orders along the $b$-axis, since (010) reflection (Fig. 1b) does not gain the intensity within the experimental error.

In conclusion, for the first time the magnetic structure of $\mathrm{NdMnO}_{3+\delta}$ was studied on a single crystal. Our investigation confirmed results, which have been already obtained by neutron powder diffraction [5]. The antiferromagnetic ordering, which was observed below the Néel temperature $T_{N}=85.5 \mathrm{~K}[6]$, can be attributed to the ordering of Mn sublattice. The order-to-order magnetic phase transition was observed below $25 \mathrm{~K}$. This transi-

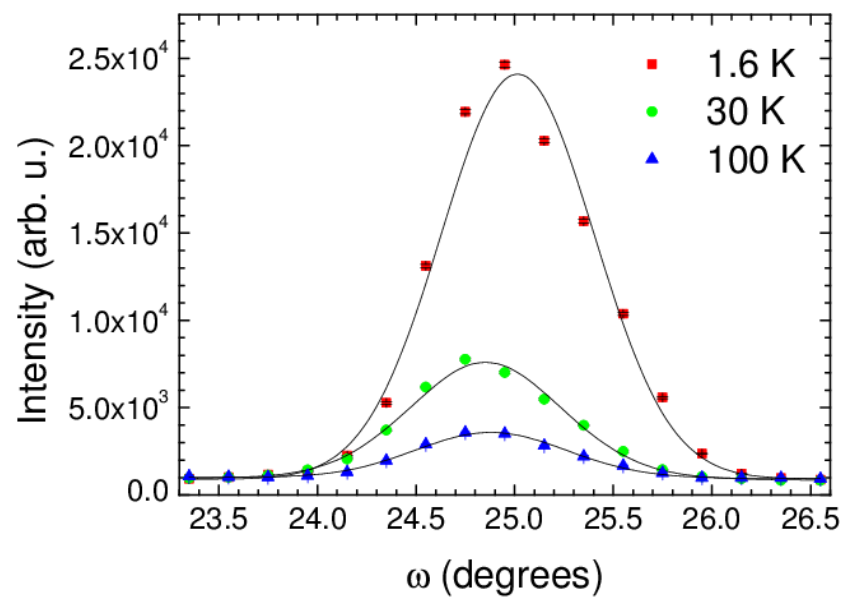

Fig. 2. The $\omega$ scans through the (200) reflection at various temperatures. The lines are the best fits using the Gaussian function.

tion we attribute to the ordering of Nd sublattice with magnetic moments oriented along the $b$-axis.

\section{Acknowledgments}

This work was supported by the European Commision under the $7^{\text {th }}$ Framework Programme through the "Research Infrastructures" action of the "Capacities" Programme, CP-CSA-INFRA-2011-1.1.17 Number 233883 NMI3 II. This work was also supported by VEGA2/178/13 and ERDF EU under the contract No. ITMS26220120047 and No. ITMS26110230034.

\section{References}

[1] H. Kuwahara, K. Noda, J. Nagayama, S. Nakamura, Phys. B 359 - 361, 1279 (2005).

[2] F. Bartolomé, J. Bartolomé, J. Campo, Phys. B $312-$ 313, 769 (2002).

[3] F. Bartolomé, J. Herrero-Albillos, L.M. García, J. Bartolomé, N. Jaouen, A. Rogalev, J. Appl. Phys. 97, 10A503 (2005).

[4] X. Moya, L.E. Hueso, F. Maccherozzi, A.I. Tovstolytkin, D.I. Podyalovskii, C. Ducati, L.C. Phillips, M. Ghidini, O. Hovorka, A. Berger, M.E. Vickers, E. Defay, S.S. Dhesi, N.D. Mathur, Nat. Mater. 12, 52 (2013).

[5] A. Muñoz, J.A. Alonso, M.J. Martínez-Lope, J.L. García-Muñoz, M.T. Fernández-Díaz, J. Phys.: Condens. Matter 12, 1361 (2000).

[6] M. Mihalik jr., M. Mihalik, J. Lazúrová, M. Fitta, M. Vavra, EPJ Web of Conferences 40, 15007 (2013).

[7] I.O. Troyanchuk, V.A. Khomchenko, G.M. Chobot, A.I. Kurbakov, A.N. Vasil'ev, V.V. Eremenko, A.V. Sirenko, M.Yu. Shvedun, H. Szymczak, R. Szymczak, J. Phys.: Condens. Matter 15, 8865 (2003). 